\title{
Seesaw Neutrinos from the Heterotic String
}

\author{
Wilfried Buchmüller, ${ }^{1}$ Koichi Hamaguchi, ${ }^{2}$ Oleg Lebedev, ${ }^{3}$ Saúl Ramos-Sánchez, ${ }^{4}$ and Michael Ratz \\ ${ }^{1}$ Deutsches Elektronen-Synchrotron DESY, 22603 Hamburg, Germany \\ ${ }^{2}$ Department of Physics, University of Tokyo, Tokyo 113-0033, Japan \\ ${ }^{3}$ CERN, Theory Division, CH-1211 Geneva 23, Switzerland \\ ${ }^{4}$ Physikalisches Institut der Universität Bonn, Nussallee 12, 53115 Bonn, Germany \\ ${ }^{5}$ Physik Department T30, Technische Universität München, James-Franck-Strasse, 85748 Garching, Germany
}

(Received 9 March 2007; published 9 July 2007)

\begin{abstract}
We study the possibility of realizing the neutrino seesaw mechanism in the $E_{8} \times E_{8}$ heterotic string. In particular, we consider its $\mathbb{Z}_{6}$ orbifold compactifications leading to the supersymmetric standard model gauge group and matter content. We find that these models possess all the necessary ingredients for the seesaw mechanism, including the required Dirac Yukawa couplings and large Majorana mass terms. We argue that this situation is quite common in heterotic orbifolds. In contrast with the conventional seesaw of grand unified theories (GUTs), no large GUT representations are needed to generate the Majorana mass terms. The total number of right-handed neutrinos can be very large, up to $\mathcal{O}(100)$.
\end{abstract}

DOI: 10.1103/PhysRevLett.99.021601

The seesaw mechanism [1-3] is perhaps the most attractive way to explain the smallness of the neutrino masses. Its essential ingredients are heavy Majorana neutrinos and their Yukawa couplings to the left-handed neutrinos. The supersymmetric seesaw mechanism is described by the superpotential

$$
W=Y_{\nu}^{i j} \phi^{u} \ell_{i} N_{j}+\frac{1}{2} M_{j k} N_{j} N_{k},
$$

where $\phi^{u}$ and $\ell_{i}(i=1,2,3)$ are the Higgs and lepton doublets, and $N_{j}(1 \leq j \leq n)$ are some heavy standard model singlets. At low energies, this leads to 3 light neutrinos with masses of order $\left(Y_{\nu}\left\langle\phi^{u}\right\rangle\right)^{2} / M$, where $Y_{\nu}$ and $M$ represent typical values of $Y_{\nu}^{i j}$ and $M_{j k}$, respectively. For $Y_{\nu} \sim 1$ and $M \sim 10^{16} \mathrm{GeV}$, one has $m_{\nu} \sim 10^{-3} \mathrm{eV}$. The scales of the atmospheric and solar neutrino oscillations, $\sqrt{\Delta m_{\mathrm{atm}}^{2}} \simeq 0.04 \mathrm{eV}$ and $\sqrt{\Delta m_{\mathrm{sol}}^{2}} \simeq 0.008 \mathrm{eV}$ [4], are suspiciously close to this scale. This hints at grand unified theory (GUT) structures behind the seesaw.

In conventional GUTs, $N_{j}$ are members of GUT matter multiplets, e.g., a 16-plet of $\mathrm{SO}(10)$, and $M_{j k}$ are related to a vacuum expectation value (VEV) of a large GUT representation, e.g., a $\overline{\mathbf{1 2 6}}$-plet of $\mathrm{SO}(10)$. In this case the Majorana mass terms originate from the coupling $1616 \overline{126}$ (cf. [5]).

Difficulties arise when one attempts to embed this attractive mechanism in string theory [6], which is the best candidate for the ultraviolet completion of the standard model. One of the problems is that string GUTs usually do not allow for large GUT representations such as $\overline{\mathbf{1 2 6}}$. Other obstacles are posed by string selection rules for the superpotential couplings which forbid some of the necessary couplings in certain models [7]. For some string models one can obtain Dirac neutrino masses [8,9]. Recently, large Majorana masses have been obtained from instanton ef-
PACS numbers: 11.25.Wx, 11.25.Mj, 12.60.Jv, 14.60.Pq

fects [10-12] as well as from higher order operators [13] and small Majorana masses from large volume compactifications [14]. However, it turned out to be very difficult to obtain the standard seesaw mechanism in consistent string models which lead to the gauge group and the matter content of the minimal supersymmetric standard model (MSSM).

The purpose of this Letter is to show that these problems can be circumvented in the $E_{8} \times E_{8}$ heterotic string $[15,16]$. We base our study on orbifold compactifications of the heterotic string [17-21]. Recent work on an orbifold GUT interpretation of heterotic models [22-24] has triggered renewed interest in these constructions. It has led to the idea of stringy local grand unification [25-28], which allows for an intuitive explanation of the multiplet structure of the standard model as well as gauge coupling unification.

The key point of local grand unification is that fields localized in the compact space at points with a GUT symmetry appear as complete representations of that GUT in the low-energy spectrum. This applies, in particular, to 16-plets of local SO(10) GUTs. On the other hand, the low-energy gauge group is an intersection of various local GUTs in $E_{8} \times E_{8}$ and can be just the standard model gauge group. It has been shown that, in the context of the $\mathbb{Z}_{6}$-II orbifold, the concept of local grand unification facilitates construction of phenomenologically promising models which admit vacua with the exact matter content of the MSSM $[25,28,29]$. These models inherit certain features of the underlying GUTs such as the GUT structure of matter multiplets and the standard GUT hypercharge. There are also significant differences from the usual 4D GUTs. Most importantly, the bulk and higher twisted sector states appear in split multiplets, thus allowing for natural doublettriplet splitting. Furthermore, mixing of the localized gen- 
erations with vectorlike bulk states breaks the unwanted GUT relations for the fermion masses $[28,30]$.

The Yukawa couplings do not necessarily preserve the symmetry of local GUTs. The symmetry of the nonlocal coupling in 10D is an intersection of the local gauge groups at the vertices (Fig. 1). This implies, for example, that the Majorana mass terms for the neutrino components of the 16-plets can originate from the coupling

$$
\nu_{16} \times \nu_{16} \times(\mathrm{SM} \text { singlets }),
$$

where the singlets belong neither to $\stackrel{(-)}{\mathbf{1 6}}$ nor to $\overrightarrow{\mathbf{1 2 6}}$ of $\mathrm{SO}(10)$. Furthermore, any SM singlet can play the role of the right-handed neutrino as long as it has a Yukawa coupling to the lepton doublets and a large Majorana mass. These are abundant in orbifold models and typical models contain $\mathcal{O}(100)$ such singlets.

Heterotic orbifold models are defined by the orbifold twist $v$, torus lattice and the gauge backgrounds which include the gauge shift $V$ and the Wilson lines $W$. With an appropriate choice of these quantities, one can obtain models with (i) the standard model gauge group $G_{\mathrm{SM}}$ times extra group factors and (ii) 3 matter generations, 2 Higgs doublets, plus extra vectorlike states [25,29]. Such models contain an "anomalous" U(1) which induces the FayetIliopoulos (FI) $D$ term,

$$
D_{\text {anom }}=\frac{g M_{\mathrm{Pl}}^{2}}{192 \pi^{2}} \operatorname{Tr} \mathrm{t}_{\mathrm{anom}}+\sum_{i} q_{i}\left|\phi_{i}\right|^{2},
$$

where $t_{\text {anom }}$ is the anomalous $\mathrm{U}(1)$ generator, $q_{i}$ are the anomalous charges of fields $\phi_{i}$, and $g$ is the gauge coupling. This triggers spontaneous gauge symmetry breaking while preserving supersymmetry [31]. Some of the fields charged under the anomalous U(1) (and, in addition, under other gauge groups) develop nonzero VEVs thereby reducing gauge symmetry. The scale of these VEVs is set by the FI term which is somewhat below the string scale. This eventually determines the seesaw scale. In general, any SM singlets can get large VEVs as long as it is consistent with

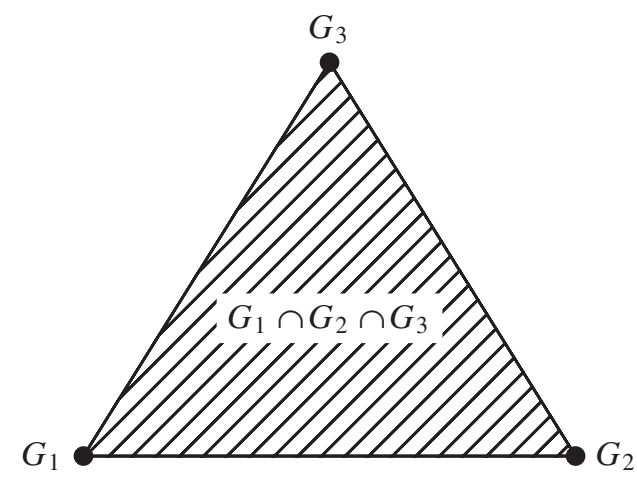

FIG. 1. Couplings involving fields localized at different fixed points in $10 \mathrm{D}$ preserve the symmetry corresponding to the intersection of the local gauge groups $G_{i}$. supersymmetry, and one can obtain the standard model gauge symmetry times that of the hidden sector,

$$
G \rightarrow \mathrm{SU}(3)_{c} \times \mathrm{SU}(2)_{L} \times \mathrm{U}(1)_{Y} \times G_{\text {hidden }} .
$$

The singlet VEVs are not necessarily associated with flat directions in the field space and generally correspond to isolated solutions to supersymmetry equations [28]. The hidden matter gauge group $G_{\text {hidden }}$ can be responsible for spontaneous supersymmetry breaking. In fact, within the class of models with the MSSM spectrum, gaugino condensation in the hidden sector favors $\mathrm{TeV}$-scale soft masses for the observable fields [32].

The nonzero singlet VEVs lead to the mass terms for the vectorlike states,

$$
W=x_{i} \bar{x}_{j}\left\langle s_{a} s_{b} \ldots\right\rangle,
$$

where $x_{i}, \bar{x}_{j}$ are the vectorlike exotics and $\left\langle s_{k}\right\rangle$ are the SM singlet VEVs in string units. Such a coupling must be consistent with the string selection rules $[33,34]$. It has been shown that many $\mathbb{Z}_{6}$-II models satisfy this requirement and all of the vectorlike exotics can be decoupled [29]. This results in the MSSM spectrum at low energies.

Similarly, the singlet VEVs induce "Majorana" mass terms for the SM singlets as well as the neutrino Yukawa couplings of Eq. (1),

$$
M_{i j} \sim\left\langle s_{a} s_{b} \ldots\right\rangle, \quad Y_{\nu}^{i j} \sim\left\langle s_{\alpha} s_{\beta} \ldots\right\rangle,
$$

as long as it is consistent with string selection rules.

Identification of right-handed neutrinos is intimately related to the issue of baryon/lepton number violation. In generic vacua, any SM singlet can play the role of the righthanded neutrino. However, such vacua also suffer from excessive $R$-parity violating interactions. The simplest way to suppress these interactions is to identify a $B-L$ gauge symmetry and enforce either its approximate conservation or conservation of its discrete ("matter parity") subgroup. In local GUTs, the $B-L$ generator resembles the standard GUT $B-L$, but also requires extra U(1) components beyond $\mathrm{SO}(10)$. It is nonanomalous and produces the standard $B-L$ charges for the SM matter. If $B-L$ is broken by VEVs of fields carrying even charges under $B-$ $L$, the matter parity $(-1)^{3(B-L)}$ is conserved. This forbids dangerous $R$-parity violating interactions and requires the right-handed neutrino to carry the charge $q_{B-L}= \pm 1$. Another possibility is that $\mathrm{U}(1)_{B-L}$ is broken at an intermediate scale $M_{B-L}$ such that all $R$-parity violating couplings are suppressed by $M_{B-L} / M_{\mathrm{Pl}}$. In this case, Majorana mass terms for the right-handed neutrinos are allowed only upon $B-L$ breaking, which lowers the seesaw scale to intermediate energies. In what follows, we consider these possibilities in specific heterotic orbifold models.

Model 1 [29]. - It is a $\mathbb{Z}_{6}$-II orbifold based on a Lie torus lattice $\mathrm{G}_{2} \times \mathrm{SU}(3) \times \mathrm{SO}(4)$ with a twist vector $v_{6}=$ $\left(\frac{1}{6}, \frac{1}{3},-\frac{1}{2}\right)$ (see [28] for details). The gauge shift in an orthonormal $E_{8} \times E_{8}$ basis is given by 


$$
V=\left(\frac{1}{3},-\frac{1}{2},-\frac{1}{2}, 0^{5}\right)\left(\frac{1}{2},-\frac{1}{6},\left(-\frac{1}{2}\right)^{5}, \frac{1}{2}\right),
$$

while the Wilson lines are chosen as

$$
\begin{aligned}
& W_{2}=\left(\frac{1}{4},\left(-\frac{1}{4}\right)^{4},\left(\frac{1}{4}\right)^{3}\right)\left(1,-1,-\frac{5}{2},-\frac{3}{2},-\frac{1}{2},-\frac{5}{2},-\frac{3}{2}, \frac{3}{2}\right), \\
& W_{3}=\left(-\frac{1}{2},-\frac{1}{2},\left(\frac{1}{6}\right)^{6}\right)\left(\frac{10}{3}, 0,-6,-\frac{7}{3},-\frac{4}{3},-5,-3,3\right) .
\end{aligned}
$$

Here the superscripts denote repeated entries. The gauge group after compactification is $G_{\mathrm{SM}} \times \mathrm{SO}(8) \times \mathrm{SU}(2) \times$ $\mathrm{U}(1)^{7}$, while the matter content is that of the MSSM plus vectorlike exotics. The $B-L$ generator is identified with

$$
\mathrm{t}_{B-L}=\left(1,1,0,0,0,-\frac{2}{3},-\frac{2}{3},-\frac{2}{3}\right)\left(\frac{1}{2}, \frac{1}{2}, 0, \frac{1}{2}, \frac{1}{2}, 0,0,0\right) .
$$

In general supersymmetric configurations, many SM singlets get nonzero VEVs. Choosing a subset of such singlets with 0 or $\pm 2 B-L$ charges, the unbroken gauge symmetry is

$$
G_{\mathrm{SM}} \times G_{\text {hidden }},
$$

where $G_{\text {hidden }}=\mathrm{SO}(8)$, while all of the exotic states get large masses and decouple. This vacuum preserves the matter parity $(-1)^{3(B-L)}$.

We find that there are 39 right-handed neutrinos defined by $q_{B-L}= \pm 1$, two of which are members of the localized 16-plets. They have Yukawa couplings to the lepton doublets and large Majorana mass terms. We have calculated the $3 \times 39$ Yukawa matrix $Y_{\nu}$ and $39 \times 39$ Majorana mass matrix $M$ of Eq. (1) up to 6th order in the singlet VEVs. That is, for each matrix element, we have determined at which order in the superpotential a nonzero coupling is allowed by string selection rules. Each entry depends on the quantum numbers and the localization of the Majorana neutrinos and involves products of different singlets and moduli-dependent Yukawa couplings. We then assume that the main hierarchy in these entries comes from products of singlet VEVs so that these matrices can be treated as textures. The effective mass matrix for the left-handed neutrinos,

$$
M_{\mathrm{eff}}=-v_{u}^{2} Y_{\nu} M^{-1} Y_{\nu}^{T}
$$

with $v_{u}$ being the up-type Higgs VEV, can be represented by the texture

$$
M_{\mathrm{eff}} \sim-\frac{v_{u}^{2}}{M_{*}}\left(\begin{array}{ccc}
1 & s & s \\
s & s^{2} & s^{2} \\
s & s^{2} & s^{2}
\end{array}\right) .
$$

Here $s<1$ represents a generic singlet VEV in string units and $M_{*}$ is the effective seesaw scale. $Y_{\nu}$ contains entries with powers of $s$ between 1 and 5, while the dependence of the eigenvalues of $M$ ranges from $s$ to $s^{8}$ (with no massless eigenstates at generic points in moduli space). This results in a strong $s$ dependence of the effective seesaw scale $M_{*}$. This scale is further suppressed by the large multiplicity of heavy singlets $N, M_{*} \propto N^{-x}$ with $0<x<2$. The value of $x$ depends on the texture. For example, when all the sin- glets contribute equally, $x=2$, whereas $x=0$ if only a fixed number of neutrinos have non-negligible couplings. For the present model, we find

$$
M_{*} \sim 0.1 s^{5} M_{\mathrm{str}} \sim 10^{14} \mathrm{GeV},
$$

for the string scale $M_{\text {str }}=2 \times 10^{17} \mathrm{GeV}$ and $s \sim 0.3$. The obtained texture (9) is of course model dependent.

The corresponding charged lepton Yukawa matrix is of the form

$$
Y_{e} \sim\left(\begin{array}{ccc}
1 & 1 & c \\
c & c & c^{2} \\
0 & 0 & 0
\end{array}\right),
$$

where " 0 " denotes absence of the coupling up to 6th order in the singlet VEVs $c$. Such zeros are expected to be filled in at higher orders. Here we are again using a single expansion parameter although in practice there are many variables.

These crude estimates show that reasonable fermion masses can in principle be obtained. Inserting order one coefficients in the textures, one finds that the eigenvalues scale as

$$
m_{\nu_{i}} \sim\left(1, s^{2}, s^{2}\right) \frac{v_{u}^{2}}{M_{*}}, \quad m_{e_{i}} \sim(1, c, 0) v_{d},
$$

where $v_{d}$ is the down-type Higgs VEV. For $s \sim 0.3$ and $c \sim 0.1$ the textures reproduce roughly the observed lepton mass hierarchy. The above texture favors the normal neutrino mass hierarchy and can accommodate small and large mixing angles. Further details of the model are available at [35].

Model 2 [25]. - It is based on the same orbifold with a different choice of the gauge backgrounds. The gauge shift and the Wilson lines are

$$
\begin{aligned}
V & =\left(\frac{1}{2}, \frac{1}{2}, \frac{1}{3}, 0,0,0,0,0\right)\left(\frac{1}{3}, 0,0,0,0,0,0,0\right), \\
W_{2} & =\left(\frac{1}{2}, 0, \frac{1}{2}, \frac{1}{2}, \frac{1}{2}, 0,0,0\right)\left(-\frac{3}{4}, \frac{1}{4}, \frac{1}{4},-\frac{1}{4}, \frac{1}{4}, \frac{1}{4}, \frac{1}{4},-\frac{1}{4}\right), \\
W_{3} & =\left(\frac{1}{3}, 0,0, \frac{1}{3}, \frac{1}{3}, \frac{1}{3}, \frac{1}{3}, \frac{1}{3}\right)\left(1, \frac{1}{3}, \frac{1}{3}, \frac{1}{3}, 0,0,0,0\right) .
\end{aligned}
$$

The $B-L$ generator is given by

$$
\mathrm{t}_{B-L}=\left(0,1,1,0,0,-\frac{2}{3},-\frac{2}{3},-\frac{2}{3}\right)\left(\frac{1}{2}, \frac{1}{2}, \frac{1}{2},-\frac{1}{2}, 0,0,0,0\right) .
$$

This model also produces the exact MSSM gauge group and spectrum (up to the hidden sector) in generic SUSY vacua where many SM singlets get large VEVs. Interestingly, there are $21 \mathrm{SM}$ singlets with $q_{B-L}=+1$ and 18 singlets with $q_{B-L}=-1$. We found that their $21 \times$ 18 mass matrix $M_{i j}$ has the full rank once singlets with $q_{B-L}=0$ develop VEVs. Thus, there are exactly three (massless) right-handed neutrinos with $q_{B-L}=+1$ in the limit of $\mathrm{U}(1)_{B-L}$ conservation.

Unlike model 1 , model 2 does not have a natural $R$ or matter parity once the $\mathrm{U}(1)_{B-L}$ symmetry is broken. This is because there are no singlets with even nonzero $q_{B-L}$. The simplest way to suppress $R$-parity violation is to break 
$\mathrm{U}(1)_{B-L}$ at an intermediate scale. This occurs when the SM singlets charged under $B-L$ develop hierarchically small VEVs, $M_{B-L} \ll M_{\text {str. }}$. Although the details of this mechanism are not clear, this scale can, in principle, be generated dynamically and may be related to nonperturbative dynamics such as formation of condensates.) The three righthanded neutrinos with $q_{B-L}=1$, which remain massless under the conserved $B-L$, now obtain masses of order $M_{B-L}^{2} / M_{\text {str }}$ via VEVs of singlets with $q_{B-L}=-1$. They contribute to the neutrino masses as

$$
\Delta m_{\nu} \sim v_{u}^{2} \frac{y^{2}}{M_{B-L}{ }^{2} / M_{\mathrm{str}}},
$$

where $y$ is the corresponding $B-L$ conserving Yukawa coupling.

Furthermore, states with $q_{B-L} \neq 1$ can also play the role of right-handed neutrinos. The corresponding Yukawa couplings violate $B-L$ and are suppressed by $M_{B-L} / M_{\text {str }}$. Their contribution is

$$
\Delta m_{\nu} \sim v_{u}^{2} \frac{\left(M_{B-L} / M_{\mathrm{str}}\right)^{2}}{\tilde{M}},
$$

where $\tilde{M}$ is the corresponding Majorana mass.

Comparing Eqs. (13) and (14), we see that the first contribution induces too large neutrino masses, unless $y$ is suppressed, while the second contribution is very small, unless $\tilde{M}$ is suppressed. Such suppression can come about when the relevant couplings are only allowed at high orders by string selection rules or if the relevant singlet VEVs are very small. We have calculated Yukawa couplings $y$ for a class of vacua in model 2. Although the necessary ingredients for the seesaw are present, we have not been able to identify a natural suppression mechanism for $y$ when one links suppression of $R$-parity violation to an approximate $\mathrm{U}(1)_{B-L}$. The solution is likely to require going beyond our crude approximation that many different singlets attain similar VEVs.

Our main conclusion is that heterotic orbifolds allow for many realistic vacua with the massless spectrum of the MSSM [29], which also have the required ingredients for the seesaw. In such vacua, many SM singlets get large VEVs consistent with supersymmetry (not necessarily along flat directions). This generates Majorana masses for abundant SM singlets, which play the role of the right-handed neutrinos. The effective seesaw scale is model dependent, but typically somewhat below the string scale. In models with local GUTs, this scale is intimately related to $B-L$ breaking. Although the structure of matter multiplets is similar to that of 4D GUTs, no large GUT representations are required to generate Majorana mass terms. As a result, potentially realistic neutrino mass patterns can be obtained.

This research was supported by the DFG cluster of excellence Origin and Structure of the Universe, the
European Union 6th framework Program No. MRTN-CT2004-503069 "Quest for Unification", No. MRTN-CT2004-005104 "ForcesUniverse", MRTN-CT-2006035863 "UniverseNet" and SFB-Transregios 27 "Neutrinos and Beyond" and 33 "The Dark Universe" by Deutsche Forschungsgemeinschaft (DFG).

[1] P. Minkowski, Phys. Lett. 67B, 421 (1977).

[2] T. Yanagida, KEK Report No. 79-18, 1979, p. 95.

[3] M. Gell-Mann, P. Ramond, and R. Slansky, in Supergravity, edited by $\mathrm{P}$. van Nieuwenhuizen and D. Freedman (North Holland, Amsterdam, 1979), p. 315.

[4] M. Maltoni et al., New J. Phys. 6, 122 (2004).

[5] R. N. Mohapatra and A. Y. Smirnov, Annu. Rev. Nucl. Part. Sci. 56, 569 (2006).

[6] E. Witten, Nucl. Phys. B268, 79 (1986).

[7] J. Giedt et al., Phys. Rev. D 71, 115013 (2005).

[8] L. E. Ibáñez, F. Marchesano, and R. Rabadan, J. High Energy Phys. 11 (2001) 002.

[9] I. Antoniadis et al., Nucl. Phys. B660, 81 (2003).

[10] R. Blumenhagen, M. Cvetič, and T. Weigand, Nucl. Phys. B771, 113 (2007).

[11] L. E. Ibáñez and A. M. Uranga, J. High Energy Phys. 03 (2007) 052.

[12] M. Cvetic, R. Richter, and T. Weigand, arXiv:hep-th/ 0703028.

[13] J.E. Kim, Phys. Lett. B 591, 119 (2004).

[14] J.P. Conlon and D. Cremades, arXiv:hep-ph/0611144 [Phys. Rev. Lett. (to be published)].

[15] D. J. Gross et al., Phys. Rev. Lett. 54, 502 (1985).

[16] D. J. Gross et al., Nucl. Phys. B256, 253 (1985).

[17] L. J. Dixon et al., Nucl. Phys. B261, 678 (1985).

[18] L. J. Dixon et al., Nucl. Phys. B274, 285 (1986).

[19] L. E. Ibáñez, H.P. Nilles, and F. Quevedo, Phys. Lett. B 187, 25 (1987).

[20] L. E. Ibáñez et al., Phys. Lett. B 191, 282 (1987).

[21] J. A. Casas, E. K. Katehou, and C. Muñoz, Nucl. Phys. B317, 171 (1989).

[22] T. Kobayashi, S. Raby, and R. J. Zhang, Phys. Lett. B 593, 262 (2004).

[23] S. Förste et al., Phys. Rev. D 70, 106008 (2004).

[24] T. Kobayashi, S. Raby, and R. J. Zhang, Nucl. Phys. B704, 3 (2005).

[25] W. Buchmüller et al., Phys. Rev. Lett. 96, 121602 (2006).

[26] W. Buchmüller et al., Nucl. Phys. B712, 139 (2005).

[27] W. Buchmüller et al., arXiv:hep-ph/0512326.

[28] W. Buchmüller et al., arXiv:hep-th/0606187.

[29] O. Lebedev et al., Phys. Lett. B 645, 88 (2007).

[30] T. Asaka, W. Buchmüller, and L. Covi, Phys. Lett. B 563, 209 (2003).

[31] M. Dine, N. Seiberg, and E. Witten, Nucl. Phys. B289, 589 (1987).

[32] O. Lebedev et al., Phys. Rev. Lett. 98, 181602 (2007).

[33] S. Hamidi and C. Vafa, Nucl. Phys. B279, 465 (1987).

[34] L. J. Dixon et al., Nucl. Phys. B282, 13 (1987).

[35] K. Hamaguchi et al., einrichtungen.physik.tu-muenchen. de/T30e/seesaw/. 